\title{
Photonic Generation of Phase-Modulated RF Signals for Pulse Compression Techniques in Coherent Radars
}

\author{
Paolo Ghelfi, Filippo Scotti, Francesco Laghezza, and Antonella Bogoni
}

\begin{abstract}
A novel and flexible photonics-based scheme is proposed for generating phase-coded RF pulses suitable for coherent radar systems with pulse compression techniques. After selecting two modes from a mode-locked laser (MLL), the technique exploits an optical in-phase/quadrature modulator driven by a low-sample rate and low-noise direct digital synthesizer to modulate the phase of only one mode. The two laser modes are then heterodyned in a photodiode, and the RF pulse is properly filtered out. The scheme is experimentally validated implementing a 4-bit Barker code and a linear chirp on radar pulses with a carrier frequency of about $25 \mathrm{GHz}$, starting from an MLL at about $10 \mathrm{GHz}$. The measures of phase noise, amplitude- and phase-transients, and autocorrelation functions confirm the effectiveness of the scheme in producing compressed radar pulses without affecting the phase stability of the optically generated high-frequency carriers. An increase in the radar resolution from 150 to $37.5 \mathrm{~m}$ is calculated. The proposed scheme is capable of flexibly generating software-defined phase-modulated RF pulses with high stability, even at very high carrier frequency, using only a single commercial device with potentials for wideband modulation. It can therefore allow a new generation of high-resolution coherent radars with reduced complexity and cost.
\end{abstract}

Index Terms-Chirp, coherent radar, laser mode locking, microwave photonics, phase modulation.

\section{INTRODUCTION}

$\mathbf{I}$ $\mathrm{N}$ modern radar systems, the requirements of resolution, sensitivity, and flexibility are pushing the development of RF signal generators with stable high-frequency carriers and reconfigurable pulse amplitude and phase, enabling the realization of software-defined coherent radar systems. In particular, a phase modulation or coding is often added to the RF pulses [1] in coherent radars: this technique, called pulse compression, increases the radar resolution by increasing the signal bandwidth, and allows to transmit long pulses minimizing the transmitted peak power.

Manuscript received October 21, 2011; revised January 23, 2012; accepted February 07, 2012. Date of publication February 13, 2012; date of current version April 06, 2012. This work was supported in part by the European projects photonic-based full digital radar (PHODIR), Pan-European photonics task force (EURO-FOS), and active plasmonics and lossless metamaterials (ACEPLAN)

P. Ghelfi, F. Scotti, and A. Bogoni are with the National Photonic Networks Laboratory, Consorzio Nazionale Interuniversitario per le Telecomunicazioni, 56124 Pisa, Italy (paolo.ghelfi@cnit.it; filippo.scotti@cnit.it; antonella.bogoni@cnit.it).

F. Laghezza was with the Consorzio Nazionale Interuniversitario per le Telecomunicazioni, 56124 Pisa, Italy. He is now with the University of Pisa, 56126 Pisa, Italy (francesco.laghezza@iet.unipi.it).

Color versions of one or more of the figures in this paper are available online at http://ieeexplore.ieee.org.

Digital Object Identifier 10.1109/JLT.2012.2187879
Nowadays, reconfigurable radar signals can be generated exploiting direct digital synthesizers (DDSs) at intermediate frequency, followed by an upconversion to the desired carrier frequency realized by means of analog mixers. State-of-the-art DDSs have a maximum frequency limited to few gigahertz, introduce quantization errors due to digital-to-analog conversion, and phase errors depending on the phase stability of their internal clock. Both these kind of errors are increasingly significant at higher signal frequencies. Moreover, the RF mixing processes of upconversion is one of the main sources of phase noise in radar transmitters due to the frequency fluctuations induced by the analog mixers or by the frequency multipliers.

In the last few years, several solutions based on photonics have been proposed for generating phase-stable high-frequency signals avoiding the noisy operation of upconversion at the radar transmitter. Simple architectures have been first envisioned based on heterodyning of two independent lasers [2], [3], but these implementations do not allow for a very stable RF generation. In order to improve RF stability, phase locking of the beating lasers is necessary, and this usually requires more complex and cumbersome setups [4]. A relatively simple technique for generating phase-locked laser lines is the mode locking of lasers [5], [6]: the intrinsic phase-locking condition of the mode-locked laser (MLL) ensures an extremely low phase noise of the generated RF signal, in particular if the MLL is driven in regenerative configuration working as an optical clock. Moreover, the possibility of selecting laser modes with variable wavelength detuning allows the flexible production of RF carriers with tunable frequency, potentially generating any multiple frequency of the MLL repetition rate.

While a lot of efforts have been spent in the photonic generation of RF carriers, only few works have been presented so far on introducing a phase modulation or a phase coding in the photonics-based radar pulse. In the first proposals, the photonics realization of phase-modulated RF signals were based on architectures realizing a wavelength-to-time conversion [7]-[11]. In these schemes, the desired RF waveform is coded on the wide spectrum of a pulsed laser by opportunely modulating its spectral components. The modulation is then transferred to the time domain by means of a dispersive element [12], and the RF signal is generated by detecting the time-modulated optical signal with a photodiode (PD) with suitable electro-optical bandwidth. With the wavelength-to-time conversion method, fine RF pulse modulations have been achieved, with cycle-tocycle phase control. Nevertheless, this technique shows several 
limitations. First of all, the RF pulse rate is given by the repetition rate of the optical pulses, and the RF pulse duration depends on the optical spectral width and on the amount of dispersion used. Only few technical realizations show the capability for reconfiguration [8]-[10], while others use fixed spectral filterings [7], [11]. Therefore, the wavelength-to-time conversion method lacks of the flexibility required in modern radar systems. Moreover, some technical solutions make use of free-space setups [8]-[10], and others exploit fiber-based interferometers [11] that must be stabilized against environmental fluctuations: thus, the amplitude and phase stability of the RF pulses generated by means of the wavelength-to-time conversion method are given by the precision of the spectrum modulation process, which can be too poor for a coherent radar system.

A second optical approach for the generation of phase-modulated RF pulses makes use of microwave photonic filters [13], [14]. This technique is based on the interference on a PD of two differently chirped and delayed optical pulses coming from the same pulse source. The obtained signal is an RF pulse whose duration depends on the optical pulsewidth and on the time delay between the two replicas. If the two pulses are both linearly chirped, as in [13], the RF pulse shows a linear chirp too. In [14], one of the chirped optical pulses is phase modulated in an electro-optical phase modulator, enabling phasecoded RF pulses. Besides the different technical solution, the approach with microwave photonic filters shows the same limitations as the wavelength-to-time conversion; in fact, it generates RF pulses whose width and repetition rate are defined by the setup parameters and cannot be flexibly changed, and shows problems of stability due to the fiber interferometers exploited.

More recently, a third approach has been presented for the photonic generation of phase-modulated RF pulses, which is based on the heterodyning of two continuous wave $(\mathrm{CW})$ lasers, one of which is controlled in phase by an electro-optical phase modulator [15], [16]. The obtained RF signal has a carrier frequency equal to the frequency difference of the two $\mathrm{CW}$ lasers, with an added phase modulation given by the electrical driving signal. The two $\mathrm{CW}$ lasers are obtained as the sidebands of a single $\mathrm{CW}$ laser modulated by RF synthesizers, so that their beating suppresses the common-mode phase noise. The necessity of phase modulating the two sidebands in two different ways requires smart setups: in [15], a polarization modulator is used after a wavelength-dependent polarization rotating stage, while in [16], a phase modulator is inserted in a fiber loop including a wavelength-specific fiber Bragg grating (FBG) and an isolator. These schemes allow the generation of RF signals with large-bandwidth reconfigurable phase modulation, but using large and complex setups. Moreover, in these schemes, the phase stability of the RF carrier depends on the quality of the RF signal generator exploited, which is usually poor and tends to worsen with the carrier frequency.

In this paper, we propose a new solution for generating phase-modulated RF signals suitable for pulse compression technique in coherent radar systems. The technique exploits the heterodyning of two modes from an MLL in order to generate an RF carrier with high phase stability. The pair of modes is then easily processed in a single commercial photonic device, namely an optical in-phase/quadrature (I/Q) modulator, also

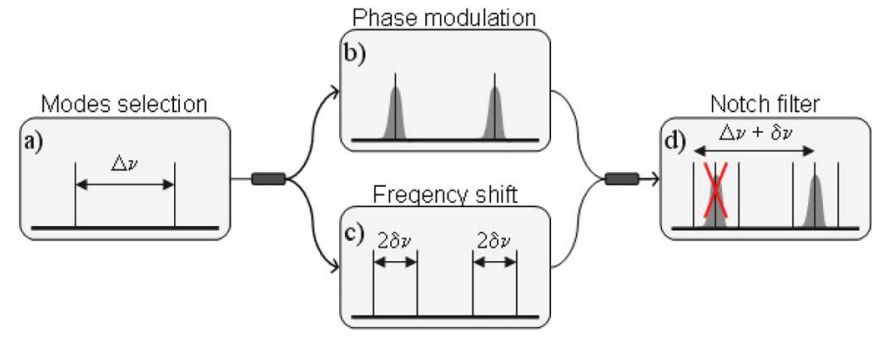

Fig. 1. Ideal scheme of principle.

referred to as a dual-parallel Mach-Zehnder (MZ) modulator, driven by a low-noise DDS. The proposed scheme is capable of generating arbitrary phase-modulated RF pulses with high stability, even at very high carrier frequency up to the extremely high frequency band $(30-300 \mathrm{GHz})$ [5], with potentials for wideband modulation.

\section{PRINCIPLE OF OPERATION}

Given two stable CW lasers, as for examples two modes of an MLL, a phase-coded RF signal is obtained if one of the CW lasers is phase modulated before they are heterodyned in a PD. To do so, the two CW lasers should be separated along different paths by splitting and filtering, in an environmentally stable ad hoc structure. A similar result can be achieved in a more flexible scheme which does not require wavelength-specific integrated components.

The scheme of principle of the proposed phase-modulated RF generation method is sketched in Fig. 1. Let us consider two modes of an MLL at frequencies $\nu_{1}$ and $\nu_{2}=\nu_{1}+\Delta \nu$, with equal amplitude. The field can be expressed as

$$
E_{a}(t)=A_{a}\left\{\exp \left[i \omega_{1} t\right]+\exp \left[i \omega_{2} t\right]\right\}
$$

where $\omega_{1}=2 \pi \nu_{1}, \omega_{2}=2 \pi \nu_{2}$, and $A$ indicates the field amplitude.

The signal is split along two different paths. In the first path, both the CW lasers are phase modulated. This can be done in a common $\mathrm{LiNbO}_{3}$ phase modulator. In the second path, they are both suppressed to generate new slightly shifted components by a carrier-suppressed amplitude modulation (AM) driven by a low-phase-noise sinusoidal signal, generating two \pm 1 -order sidebands. This operation can be realized in a common $\mathrm{LiNbO}_{3}$ MZ modulator driven in its minimum transmission point. The fields in the two paths are

$$
\begin{aligned}
E_{b}(t)= & A_{b}\left\{\exp \left[i \omega_{1} t\right]+\exp \left[i \omega_{2} t\right]\right\} \cdot \exp [i \phi(t)] \\
E_{c}(t)= & A_{c}\left\{\exp \left[i\left(\omega_{1}-\omega_{m}\right) t\right]+\exp \left[i\left(\omega_{1}+\omega_{m}\right) t\right]\right. \\
& \left.+\exp \left[i\left(\omega_{2}-\omega_{m}\right) t\right]+\exp \left[i\left(\omega_{2}+\omega_{m}\right) t\right]\right\}
\end{aligned}
$$

where $\omega_{\mathrm{m}}=2 \pi \delta \nu$ is the frequency-shifting signal. The signals $E_{b}(t)$ and $E_{c}(t)$, then, are coupled together. The resulting signal shows six spectral components. Let us suppose that we want to obtain a phase-modulated RF signal at the nominal frequency of $\Delta \nu+\delta \nu$. In the obtained signal $E_{d}(t)$, two pairs of components are at a nominal frequency difference of $\Delta \nu+\delta \nu$ (the components at $\nu_{1}$ and at $\nu_{2}+\delta \nu$, and the components at $\nu_{1}-\delta \nu$ and at $\nu_{2}$ ), and one of the components in each pair is phase modulated by $\phi(t)$ ( $\nu_{1}$ and $\nu_{2}$, respectively). In more 


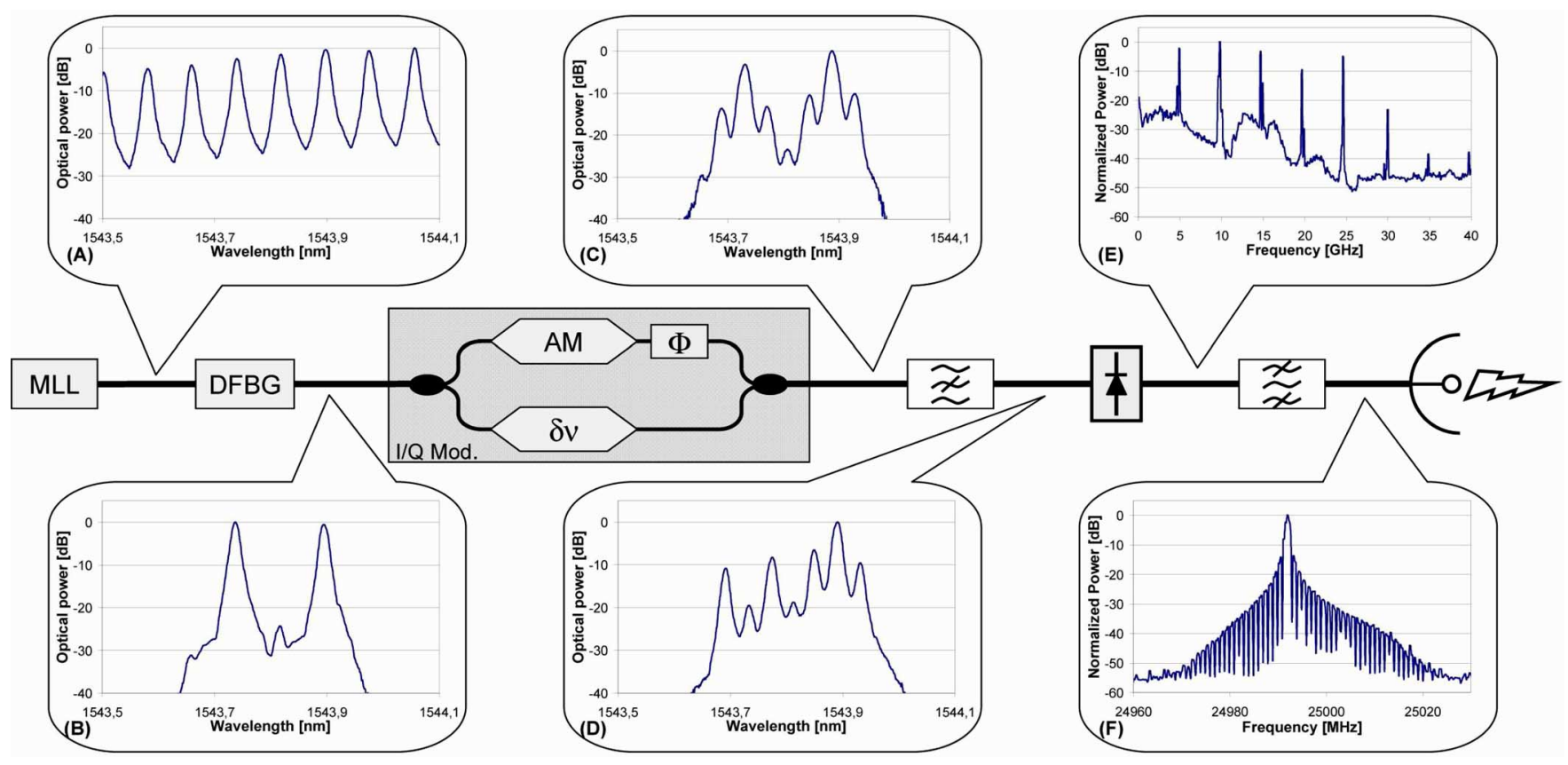

Fig. 2. Experimental setup. Insets (A)-(D) Optical spectra [resolution bandwidth (RBW) $=0.016 \mathrm{~nm}$ ] measured in different parts of the setup. Insets (E) and (F) Electrical spectra measured after the PD $(\mathrm{RBW}=3 \mathrm{MHz})$ and after the RF filter (RBW $=30 \mathrm{kHz})$, respectively.

details, the frequency difference of the components in the two pairs is $\Delta \nu+\delta \nu+\mathrm{d} \phi(t) / d t$ and $\Delta \nu+\delta \nu-\mathrm{d} \phi(t) / d t$, respectively. If these component pairs were both heterodyned in a PD, they would both generate an RF signal at a frequency carrier of $\Delta \nu+\delta \nu$, but one RF signal would be phase modulated by $\phi(t)$ while the other would be modulated by $-\phi(t)$, giving

$$
\begin{aligned}
s(t)=A_{s} \exp \left[i \omega_{\mathrm{RF}} t\right] \cdot\{\exp [i \phi(t)] & \\
& +\exp [-i \phi(t)]\}+[\ldots]
\end{aligned}
$$

where $\omega_{\mathrm{RF}}=\Delta \nu+\delta \nu=\omega_{2}-\omega_{1}+\omega_{m}$, and [...] indicates omitted terms which will be rejected by a successive RF filter. The expression (4) shows that the RF signal at $\Delta \nu+\delta \nu$ filtered out after the PD would present a modulated envelope whose shape would be given by $\cos [\phi(t)]$. Therefore, before heterodyning the entire spectrum in the $\mathrm{PD}$, one of the components in the two pairs at $\omega_{\mathrm{RF}}$ must be suppressed. This can be done by filtering out either one of the phase-modulated modes from the MLL, or one of the $\delta \nu$-shifted new components.

Considering to optically filtering out the phase-modulated laser mode at $\omega_{1}$ using a notch filter, the RF signal at the output of the PD can, therefore, be expressed as

$$
s(t)=A_{s} \exp \left\{i\left[\omega_{\mathrm{RF}} t+\phi(t)\right]\right\}+[\ldots]
$$

The expression (5) represents a phase-modulated RF signal at the desired frequency $\omega_{\mathrm{RF}}$. The same analysis can be done considering the beating at $\Delta \nu-\delta \nu$ as the nominal carrier frequency.

The operations described previously can be easily implemented exploiting a commercial optical I/Q modulator, using the bias voltage which controls the reciprocal phase shift between the two branches (usually named as Bias P), to modulate the phase of the two CW lasers. This device is an integrated structure in $\mathrm{LiNbO}_{3}$, which ensures the necessary stability against environmental fluctuations. The carrier-suppressed frequency shift on one arm of the I/Q modulator can be driven by a very stable low-frequency RF oscillator, without affecting the original reciprocal phase stability of the modes from the MLL. Moreover, the optical I/Q modulator also has an additional MZ modulator, which can be used for modulating the amplitude of the original laser components from the MLL, thus allowing the realization of phase-modulated RF radar pulses.

The proposed scheme, therefore, allows the photonics-based realization of phase-modulated RF pulses exploiting a single commercial device, with a significant simplification with respect to the previously presented photonics architectures.

\section{EXPERIMENTAL SETUP}

The experimental setup is reported in Fig. 2. The exploited laser source is a regenerative fiber MLL with a repetition rate of $9953 \mathrm{MHz}$ and a central wavelength of $1554.5 \mathrm{~nm}$. A zoom of its optical spectrum is reported in the inset (A) of Fig. 2. A dual FBG (DFBG) [17] selects two nonadjacent modes at a detuning of $20 \mathrm{GHz}$, with a suppression of $25 \mathrm{~dB}$ of the undesired adjacent modes [inset (B)]. The two selected modes are then launched into an optical I/Q modulator. This is a Covega LN86 device, with an electro-optical bandwidth of $14 \mathrm{GHz}$ for both the MZs along the two branches. Along one of the arms of the I/Q modulator, a carrier suppressed modulation is obtained by setting the relative $\mathrm{MZ}$ modulator to the minimum transmission point [18], [19]. The modulation is driven by a precise waveform synthesizer producing a sinusoidal signal at $\delta \nu=5086 \mathrm{MHz}$ with an amplitude of $0.6 \mathrm{~V}_{\pi}$, thus splitting the two modes into four new spectral components at $\pm \delta \nu$ from the original modes. The second arm of the I/Q modulator is also exploited, introducing an ON/OFF AM on the two selected modes in order to form the radar pulses. A waveform generator modulates both the laser modes with a rectangular pulse with a duration of $1 \mu$ s over a 


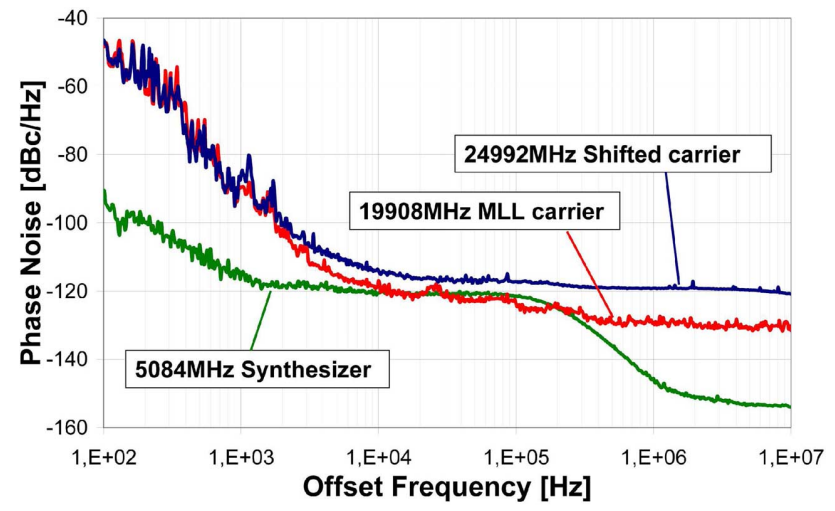

Fig. 3. Phase noise measure of the $24.992 \mathrm{GHz}$ carrier, compared with the phase noise of the nonshifted carrier from the MLL, and of the synthesizer.

period of $3 \mu \mathrm{s}$, and a modulation depth close to 1 . Finally, the reciprocal phase difference of the two modulator branches is modulated by means of a 1 Gsample/s DDS, opportunely amplified and applied to the Bias-P port of the I/Q modulator.

The optical spectrum of the output signal from the I/Q modulator is reported in the inset (C), where the four modes at $\pm 5086 \mathrm{MHz}$ from the original two laser modes are evident. In order to suppress one of the two original carriers from the MLL, an FBG with a bandwidth of $6 \mathrm{GHz}$ is used as a notch filter [see inset (D)], and the resulting signal is sent to a $50 \mathrm{GHz}$ bandwidth PD. The inset (E) shows the electrical spectrum after the PD, where several beatings take place. An RF filter centered exactly at $25000 \mathrm{MHz}$ with a bandwidth of $25 \mathrm{MHz}$ selects the beating of interest at $24992 \mathrm{MHz}$ between the nonsuppressed amplitude-modulated original laser mode and the laser line obtained by shifting the other original mode of $\delta \nu$ [see inset (F)]. The choice of a carrier frequency not perfectly aligned with the RF filter is determined by the need of keeping the RF signal below $25 \mathrm{GHz}$, in order to perform the measurements with a 50 Gsamples/s real-time oscilloscope.

\section{EXPERIMENTAL RESULTS}

Since the coherent radar systems require high phase stability, the generated signal has been first characterized in terms of phase noise. Fig. 3 shows the measured phase noise of the generated $24992 \mathrm{MHz}$ carrier, compared with the phase noise of the beating generated by the two modes from the original MLL, and with the frequency shifting signal at $5086 \mathrm{MHz}$ generated by the synthesizer. For this analysis, a CW RF carrier has been generated, turning OFF both the phase and AMs. The phase noise is measured with a signal source analyzer (SSA, Agilent E5052A) for offset frequency from $100 \mathrm{~Hz}$ - given by the common duration of a coherent radar measurement - to $10 \mathrm{MHz}$ - upper limit due to the RF filter.

The graph shows that the phase noise of the synthesizer is lower than the one of the MLL, except for the range of offset frequency from $10 \mathrm{kHz}$ to $100 \mathrm{kHz}$ where they are substantially equal. This would suggest the possibility to generate a frequency-shifted RF signal with the same noise behavior of the original MLL. Actually, Fig. 3 reports that the phase noise curve of the generated RF signal at $25 \mathrm{GHz}$ follows the behavior of the $20 \mathrm{GHz}$ signal at low offset frequency, but shows slightly higher

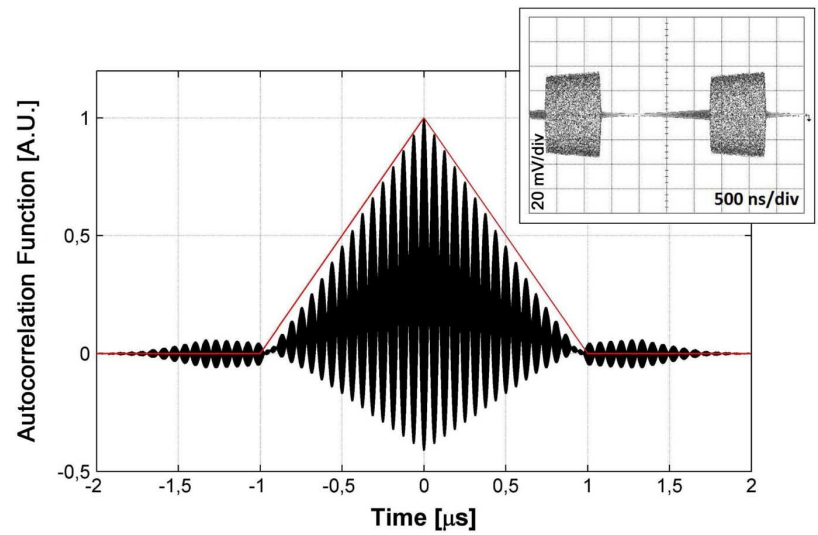

Fig. 4. Calculated AF of the optically generated RF pulse when no phase modulation is applied. The AF is compared with the AF of an ideal baseband pulse with the same duration (thin line). The inset shows the waveform of the generated RF pulse.

phase noise from $2 \mathrm{kHz}$ on, and a noise floor $10 \mathrm{~dB}$ higher for high offset frequency. We believe that this behavior is due to the added phase noise introduced by the broadband electrical amplifier exploited to boost the output signal from the PD, and could be improved using a low-noise narrow-bandwidth specific amplifier. Nevertheless, the integration of the curves in Fig. 3 over the entire offset frequency range shows that the obtained RF signal has a timing jitter of $286 \mathrm{fs}$ (equivalent to $45 \mathrm{mrad}$ at $24992 \mathrm{MHz}$ ), which is only $21 \mathrm{fs}$ higher than that of the original MLL.

The exploited MLL is a free running fiber ring laser which shows very low phase noise at high offset frequency, but is affected by mechanical vibrations that induce high phase noise at low offset frequency. It is important to note that it is possible to strongly reduce this noise if the MLL is used as an optical voltage-controlled oscillator in a phase-locked loop, where a stable low-noise reference clock is used to lock the phase of the MLL at low frequency offset. This way an extremely precise optical source can be obtained [20]. It is also interesting to note here that the MLL allows to generate RF signals over a wide frequency range by selecting modes at different detuning, without changes in the phase noise performance [5].

Once the quality of the signal is confirmed, radar pulses with no phase modulation are generated in order to be compared with the compressed ones. In Fig. 4, the autocorrelation function (AF) of the pulses is reported, calculated from a singleshot acquisition of a 50 Gsamples/s real-time oscilloscope. The receiver in a radar system operates the cross correlation between the transmitted pulse and its echo; therefore, the signal AF brings important information on the radar resolution, as will be evident in the following. In the case of noncoded pulses, the resolution is easily equal to $c T_{p} / 2$, where $c$ is the speed of light and $T_{p}$ is the radar pulse duration, which leads to a resolution of $150 \mathrm{~m}$ for the considered noncoded pulses of $1 \mu \mathrm{s}$.

In Fig. 4, the AF of the optically generated RF pulse is compared with the AF of an ideal baseband pulse of equal duration. As shown in the picture, the baseband AF fits well the envelope of the AF measured at RF, except for its minor sidelobes. These are caused by the nonideal suppression of the unwanted original mode after the optical notch filter, and by the narrow filtering of 


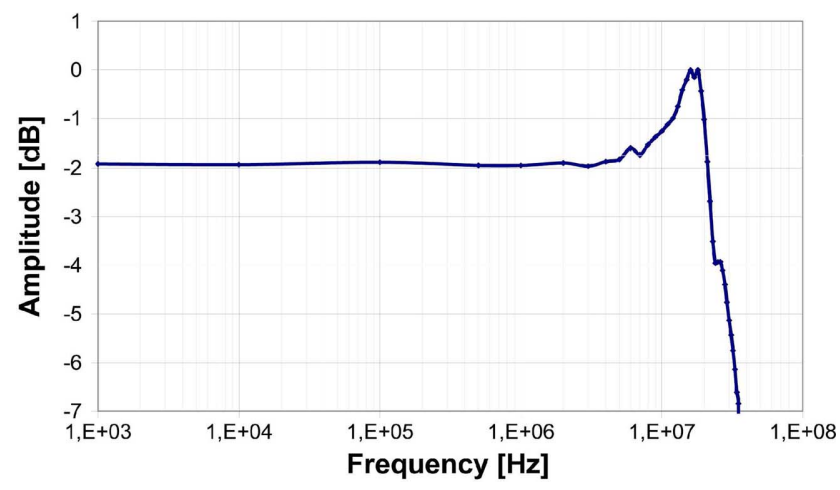

Fig. 5. Bandwidth characterization of the Bias-P port of the $\mathrm{I} / \mathrm{Q}$ modulator.

the RF signal. The effects of these nonidealities are also evident in the inset of Fig. 4 showing the RF pulse waveform.

As the $\mathrm{I} / \mathrm{Q}$ modulator is not designed to be used as a phase modulator, its characterization is necessary. In Fig. 5, the result of the characterization is plotted, realized applying a sinusoidal modulation at the Bias-P port and measuring the output AM on a $\mathrm{CW}$ laser while changing the sine frequency. The Bias-P port shows a constant frequency response up to $7 \mathrm{MHz}$ and a sharp cutoff at $20 \mathrm{MHz}$. Due to this limitation, the modulation bandwidth available with the considered device is limited to few megahertz. Nevertheless, since $40 \mathrm{GHz}$ bandwidth phase modulators are commonly implemented with state-of-the-art $\mathrm{LiNbO}_{3}$ technology, we believe that the design of a custom I/Q modulator with wideband phase modulation capability would not present technological issues.

The system is then tested applying two different phase modulations commonly used in radar applications: Barker codes and linear frequency chirp.

Barker codes are particular sequences of $N$ values $a_{j}$ in the set $(-1,+1)$ for $j=1,2, \ldots, N$, such that

$$
\left|\sum_{j=1}^{N-m} a_{j} a_{j+m} \leq 1\right|
$$

for $1 \leq m<N$. They are applied to radar pulses by dividing the pulse in $N$ subpulses of equal duration, and modulating the carrier phase in each subpulse by $0^{\circ}$ or $180^{\circ}$ according to the adopted Barker code. Thanks to the coding, this kind of radar pulses show an autocorrelation peak $N$ times narrower, and autocorrelation sidelobes $N$ times smaller [1]. This means that, by analyzing the cross correlation between the detected radar echo and the transmitted pulse, an $\mathrm{N}$-times increased resolution can be obtained with respect to the unmodulated radar pulse, with a sensitivity improvement of $\log _{10} N \mathrm{~dB}$, without reducing the pulse duration.

Fig. 6(A) reports the signal applied to the Bias-P port (upper trace) for modulating the phase, and the amplitude modulating signal applied to one of the two RF ports (lower trace). The phase-controlling signal corresponds to a 4-bit Barker code (0-0-180-0) and its amplitude is set to match the $V_{\pi}$ voltage of the device, i.e., 4.75 V. Fig. 6(B) shows the oscilloscope trace of the radar pulses, thus, generated. Amplitude discontinuities
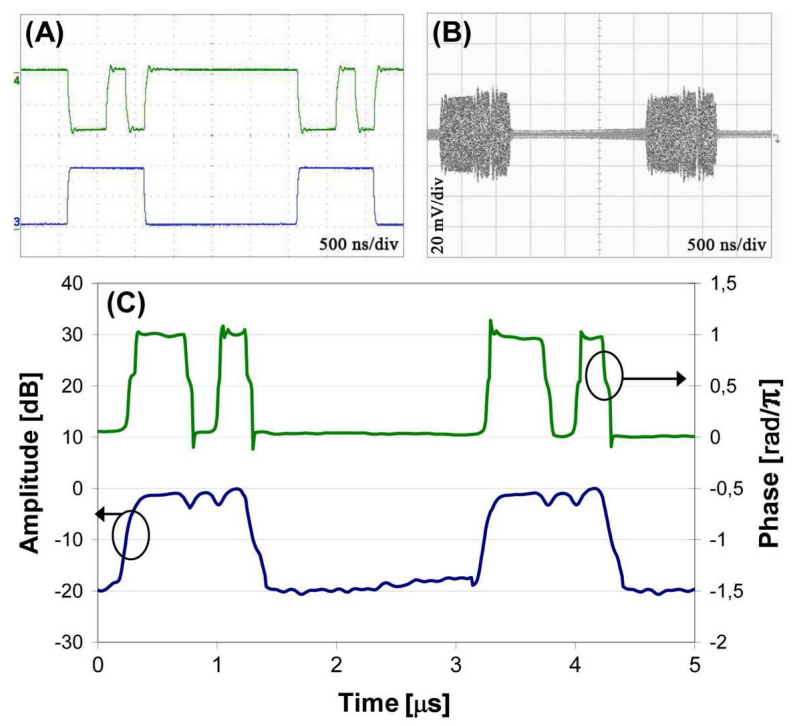

Fig. 6. Optically generated RF pulses with 4-bit Barker code. (A) Control signal for the (top) phase modulation and (bottom) AM; vertical scale $2 \mathrm{~V} /$ div. (B) Waveform of the modulated pulses. (C) Phase and amplitude transients of the signal measured with the SSA.

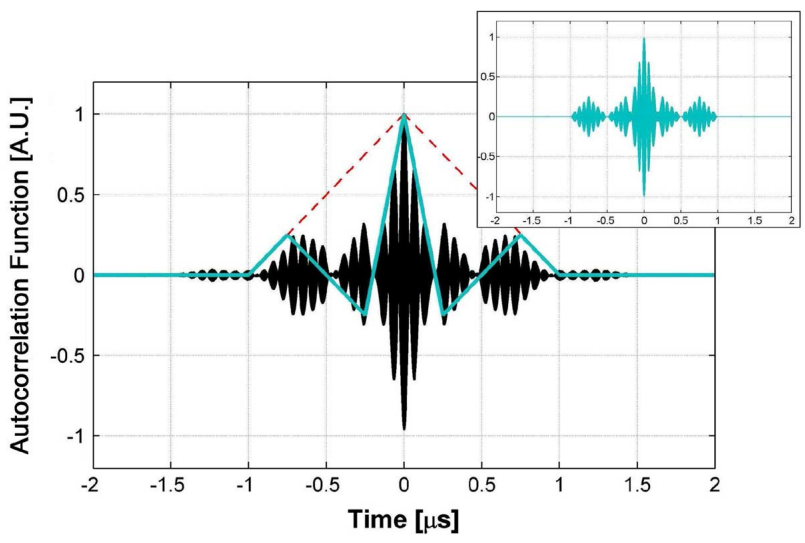

Fig. 7. AF of the Barker-coded pulses extracted from the real-time oscilloscope trace, compared with the calculated AF of a simulated coded pulse (in the inset). For the sake of clarity, the AF of the simulated baseband coded and noncoded pulses are also reported on the measured trace (solid and dashed lines, respectively).

are present at the phase jumps, and a limited pulse extinction ratio can be observed. Fig. 6(C) reports the amplitude and phase transients of the radar pulse, as acquired by the SSA. As expected, the phase modulation produces $180^{\circ}$ phase jumps in the pulses, while an amplitude extinction ratio of about $20 \mathrm{~dB}$ is measured. The amplitude transient also shows discontinuities at the phase jumps. The limited extinction ratio and the amplitude fluctuations can be ascribed to the nonideal suppression of the unwanted original mode after the notch filter, and to the narrow $\mathrm{RF}$ filtering. Those effects can, therefore, be reduced with a better design of both the optical and RF filters. Moreover, the extinction ratio of the radar pulse is generally improved by the high-power amplification stage before the radar antenna, which can ensure an extinction ratio as high as $120 \mathrm{~dB}$.

In Fig. 7, the AF of the generated Barker coded pulse is shown. As expected from the radar theory, the normalized amplitude presents sidelobe peaks at $0.25(1 / N)$ and fits well the 

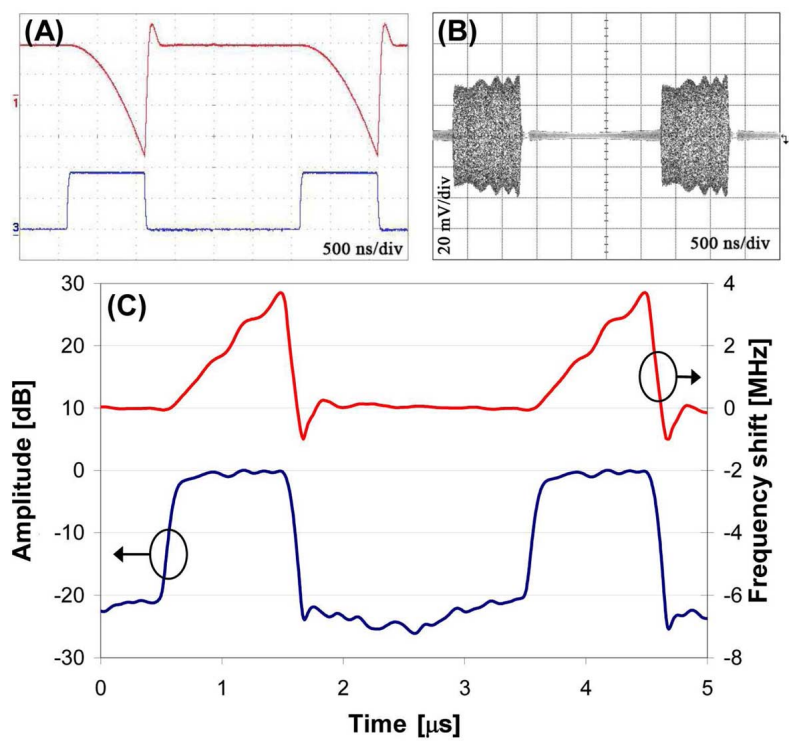

Fig. 8. Optically generated RF pulses with linear frequency chirp. (A) Control signal for the (top) phase modulation and (bottom) AM; vertical scale 5 and 2 V/div, respectively. (B) Waveform of the modulated pulses. (C) Frequency and amplitude transients of the signal measured with the SSA.

simulated envelope of the sequence. So the suitability of the proposed scheme for producing phase-coded radar signals is confirmed. Also, the duration of the AF is $0.25(1 / N)$ the one of the noncoded pulse. This means that the coding allows the same resolution of a noncoded pulse with four times shorter duration. With the particular code exploited, the range resolution is improved to $37.5 \mathrm{~m}$.

The frequency chirping is a phase-coding technique that induces a linear change in the radar carrier frequency along the pulse duration. In order to generate a linear frequency shift, a quadratic phase modulation must be applied to the carrier. The frequency deviation range is defined by following formula:

$$
\Delta f=\frac{\Delta \phi}{\pi T_{p}}=\frac{\Delta V}{V_{\pi} T_{p}}
$$

where $T_{p}$ is the pulse duration. In order to obtain a linear frequency chirp, in our experiment, the DDS is set to generate a parabola.

The parabolic phase modulation is reported in Fig. 8(A) together with the pulsed AM. The parabolic signal is designed to reach the maximum voltage variation $(18 \mathrm{~V})$ within the radar pulse duration. Since the $V_{\pi}$ of the employed Bias-P port is $4.75 \mathrm{~V}$, a linear chirp of $3.8 \mathrm{MHz}$ is expected. From the graph, elongations are also evident at the phase jumps, introduced by the electrical amplifier. Fig. 8(B) shows the waveform of the generated RF pulse, which appears to be amplitude modulated by a chirped frequency. This undesired AM is due to the nonideal suppression of the unwanted optical carrier that translates into an oscillating modulation. As stated by (4), it is proportional to the cosine of a parabola. Fig. 8(C) reports the traces of the frequency and amplitude transients, as acquired with the SSA. The measured frequency drift corresponds to the expected $3.8 \mathrm{MHz}$ and shows a linear trend. The elongations reported in Fig. 8(A) are transferred on the frequency of the radar pulse. The minor deviations from the linear behavior are probably caused by the

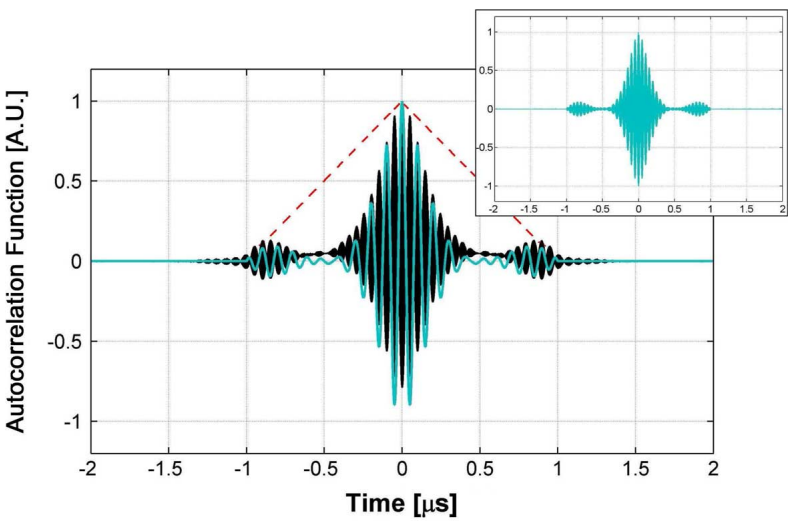

Fig. 9. AF of the chirped pulses extracted from the real-time oscilloscope trace, compared with the calculated AF of a simulated chirped pulse (in the inset). For the sake of clarity, the AFs of the simulated baseband coded and noncoded pulses are also reported on the measured trace (solid and dashed lines, respectively).

narrow and nonregular bandwidth of the modulator Bias P (see Fig. 5).

Fig. 9 shows the AF of the acquired chirped pulses, compared with the AF of a simulated ideal chirped pulse. The function calculated on the real data shows a good match with the simulated one, confirming once again the effectiveness of the proposed technique. In this case, it is possible to estimate a resolution improvement up to $39 \mathrm{~m}$.

\section{Discussions ANd Conclusions}

The aforementioned experiments demonstrate that the proposed architecture is capable of generating phase-modulated radar pulses at flexible carrier frequency exploiting a simple photonics-based setup. Besides the Barker codes and the linear frequency chirp, other pulse compression techniques would also be possible, as, for example, polyphase codes (Frank codes, complementary codes, or pseudorandom binary sequences applied to $\mathrm{CW}$ carriers) or nonlinear frequency modulations (e.g., raised cosine).

Although the experimental realization has been limited to a small modulation bandwidth, larger bandwidths and phase changes can be readily achieved by designing specific I/Q modulators exploiting the current photonics technologies, thus realizing bandwidth broadening up to some gigahertz, further increasing the effectiveness of the proposed scheme.

Moreover, the scheme can flexibly generate high-stability phase-modulated RF signals at very high carrier frequency, up to hundreds of gigahertz, with potentials also for terahertz and sub-terahertz signals generation, where the possibility of modulating the signal has not been extensively investigated yet.

In conclusion, we have proposed a novel and practical scheme for the photonics-based generation of phase-modulated RF signals, targeted to the application in coherent radars with pulse compression techniques. The scheme is based on the exploitation of a commercial device, an optical I/Q modulator, in a nonconventional fashion, and its performance has been confirmed by experimental results. The high phase stability over a broad frequency range, and the wideband modulation capability of the proposed scheme, together with its compactness 
and reliability, could allow the realization of a new generation of high frequency fully digital phase-coded radars, with wide application opportunities even in the related field of wireless communications.

\section{ACKNOWLEDGMENT}

The authors would like to thank P. Pérez-Millán, G. E. Villanueva, J. Palací, and J. L. Cruz for providing the DFBG.

\section{REFERENCES}

[1] M. L. Skolnik, Introduction to Radar Systems, 2nd ed. New York: McGraw-Hill, 1980.

[2] L. Goldberg, H. F. Taylor, J. F. Weller, and D. M. Bloom, "Microwave signal generation with injection locked laser diodes," Electron. Lett., vol. 19 , no. 13, pp. 491-493, Jun. 1983.

[3] J. Sun, Y. Dai, X. Chen, Y. Zhang, and S. Xie, "Stable dual-wavelength DFB fiber laser with separate resonant cavities and its application in tunable microwave generation," IEEE Photon. Technol. Lett., vol. 18, no. 24, pp. 2587-2589, Dec. 2006.

[4] L. Goldberg, R. D. Esman, and K. J. Williams, "Generation and control of microwave signals by optical techniques," in Proc. IEE J. Optoelectron., Aug. 1992, vol. 139, no. 4, pp. 288-295.

[5] G. Serafino, P. Ghelfi, G. E. Villanueva, J. Palací, P. Pérez-Millán, J. L. Cruz, C. Porzi, and A. Bogoni, "Stable optically generated RF signals from a fibre mode-locked laser," in IEEE Photon. Soc. Annu. Meet, Denver, CO, 2010, TuK4.

[6] T. Yilmaz, C. M. DePriest, T. Turpin, J. H. Abeles, and P. J. Delfyett, Jr. , "Toward a photonic arbitrary waveform generator using a modelocked external cavity semiconductor laser," IEEE Photon. Technol. Lett., vol. 14, no. 11, pp. $1608-1610$, Nov. 2002.

[7] B. Jalali, P. Kelkar, and V. Saxena, "Photonic arbitrary wavelength generator," in Proc. 14th Annu. Meet. Laser-Opt. Soc., Piscataway, NJ, 2001, vol. 1, pp. 253-254, Cat 01CH37242.

[8] J. D. McKinney, D. E. Leaird, and A. M. Weiner, "Millimeter-wave arbitrary waveform generation with a direct space-to-time pulse shaper," Opt. Lett., vol. 27 , no. 5, pp. 1345-1347, Aug. 2002

[9] J. Chou, Y. Han, and B. Jalali, "Adaptive RF-photonic arbitrary waveform generator," IEEE Photon. Technol. Lett., vol. 15, no. 4, pp. 581-583, Apr. 2003.

[10] I. S. Lin, J. D. McKinney, and A. M. Weiner, "Photonic synthesis of broadband microwave arbitrary waveform applicable to ultra-wideband communication," IEEE Microw. Wireless Compon. Lett., vol. 15, no. 4, pp. 226-228, Apr. 2005.

[11] H. Chi and J. P. Yao, "All-fiber chirped microwave pulse generation based on spectral shaping and wavelength-to-time conversion," IEEE Trans. Microw. Theory Tech., vol. 55, no. 9, pp. 1958-1963, Sep. 2007.

[12] Y. Park and J. Azaña, "Ultrahigh dispersion of broadband microwave signals by incoherent photonic processing," Opt. Exp., vol. 18, no. 14, pp. 14752-14761, Jul. 2010.

[13] A. Zeitouny, S. Stepanov, O. Levinson, and M. Horowitz, "Optical generation of linearly chirped microwave pulses using fiber Bragg gratings," IEEE Photon. Technol. Lett., vol. 17, no. 3, pp. 660-662, Mar. 2005.

[14] H. Chi and J. P. Yao, "An approach to photonic generation of high frequency phase-coded RF pulses," IEEE Photon. Technol. Lett., vol. 19, no. 10, pp. 768-770, May 15, 2007.

[15] H. Chi and J. P. Yao, "Photonic generation of phase-coded millimeterwave signal using a polarization modulator," IEEE Microw. Wireless Compon. Lett., vol. 18, no. 5, pp. 371-373, May 2008
[16] Z. Li, W. Li, H. Chi, X. Zhang, and J. Yao, "Photonic generation of phase-coded microwave signal with large frequency tunability," IEEE Photon. Technol. Lett., vol. 23, no. 11, pp. 712-714, Jun. 2011.

[17] P. Pérez-Millán, A. Wiberg, P. O. Hedekvist, P. A. Andrekson, and M. V. Andrésa, "Optical demultiplexing of millimeter-wave subcarriers for wireless channel distribution employing dual wavelength FBGs," Opt. Commun., vol. 275, no. 2, pp. 335-343, Jul. 2007.

[18] K. Sasagawa and M. Tsuchiya, "Low-noise and high-frequency resolution electrooptic sensing of RF near-fields using an external optical modulator," J. Lightwave Technol., vol. 26, no. 10, pp. 1242-1248, May 2008.

[19] D.-J. Lee and J. F. Whitaker, "Bandwidth enhancement of electro-optic field sensing using photonic down-mixing with harmonic sidebands," Opt. Exp., vol. 16, pp. 14771-14779, 2008

[20] L. Banchi, F. Rossi, M. Ferianis, A. Bogoni, L. Potì, and P. Ghelfi, "Synchronization of $3 \mathrm{GHz}$ repetition rate harmonically mode-locked fiber laser for optical timing applications," presented at the presented at the DIPAC Conf., Venezia, Italy, 2007.

Paolo Ghelfi received the M.S. degree in electronics engineering from the University of Parma, Parma, Italy, in 2000.

From 2000 to 2001 he was a Grantee in the Optical Communications Laboratory, Parma University. Since 2002, he has been with the National Photonic Networks Laboratory, Consorzio Nazionale Interuniversitario per le Telecomunicazioni, Pisa, Italy. His research interests include the areas of fiber optical transmissions and all-optical processing.

Filippo Scotti received the M.S. degree in physics engineering from the Politecnico of Milano, Milano, Italy, in December 2009. In 2009, he was with PGT-Photonics S.p.A, Milano, for a six-month internship.

Since 2010, he has been with the National Photonic Networks Laboratory, Consorzio Nazionale Interuniversitario per le Telecomunicazioni, Pisa, Italy. His research interests include the areas of fiber-optic transmissions with particular interest in all-optical signal processing, and ultrashort optical pulse sources and their RF applications.

Francesco Laghezza received the M.S. degree in telecommunication engineering from the University of Pisa, Pisa, Italy, in April 2009. He is currently working toward the Ph.D. degree in remote sensing at the University of Pisa, Pisa.

From 2009 to 2010, he was a Postgraduate Research Assistant at the National Interuniversity Consortium for Telecommunications. His research interests include full digital electro-optical radar design, space debris radar detection, and array processing.

Antonella Bogoni received the Ph.D. degree from the University of Parma, Parma, Italy, in 2004.

She is currently the Head of research at the National Photonic Networks Laboratory, Consorzio Nazionale Interuniversitario per le Telecomunicazioni, Pisa, Italy. Her research interests include the areas of ultrafast all-optical signal processing and digital photonics.

Dr. Bogoni received the Fulbright Advanced Research Scholarship Award for the project "Design and implementation of a $640 \mathrm{~Gb} / \mathrm{s}$ OTDM system" at the University of Southern California, CA. 